\title{
Perinatal depressive symptoms among low-income South African women at risk of depression: trajectories and predictors
}

\author{
Emily C. Garman ${ }^{1 *}$ (D), Marguerite Schneider ${ }^{1}$ and Crick Lund ${ }^{1,2}$
}

\begin{abstract}
Background: The aim of the study was to identify trajectories of perinatal depressive symptoms and their predictors among women living in a low-resource setting in South Africa, and who present with a risk of depression during pregnancy.

Methods: This is a secondary analysis of a randomised controlled trial among 384 women living in Khayelitsha, a low income setting in South Africa, recruited at their first antenatal visit if they scored 13 or above on the Edinburgh Postnatal Depression Scale, were at least 18 years of age, less than 29 weeks pregnant and spoke isiXhosa. Participants were followed up at 8 months gestation, 3 and 12 months postpartum. Latent trajectories of depressive symptoms were identified using growth mixture modelling, based on the Hamilton Depression Rating Scale (HDRS). There were no differences in HDRS scores between the control and intervention arms, so all participants were assessed together. Health, social and economic predictors of trajectories were investigated to identify high-risk groups with greater or more chronic depressive symptoms, using univariate logistic regression.

Results: Two trajectories were identified: antenatal only (91.4\%), with moderate to severe symptoms at baseline which later subside; and antenatal and postnatal (8.6\%), with severe depressive symptoms during pregnancy and later in the postpartum period, which subside temporarily to moderate levels at 3 months postpartum. Predictors for the antenatal and postnatal trajectory include severe food insecurity, intimate partner violence, lower social support, greater functional impairment, problematic drinking and suicide risk.

Conclusions: A small proportion of women who are at risk for depression antenatally remain at risk throughout the perinatal period, and can be differentiated from those who show a natural remission. Identification and referral strategies should be developed with these findings in mind, especially given the limited mental health resources in low-income settings.
\end{abstract}

Keywords: Trajectory, Depression, Perinatal, Risk factors, Low-income

\section{Background}

Depression during pregnancy and the postnatal period, known as perinatal depression, is a concern worldwide. In South Africa, the prevalence of women at high risk of depression or suffering from depression ranges between 21 and $39 \%$ antenatally [1-4], and between 16 and 32\% postnatally [5-7]. The burden of disease associated with perinatal depression and impact on child health and

\footnotetext{
* Correspondence: emily.garman@uct.ac.za

'Alan J Flisher Centre for Public Mental Health, Department of Psychiatry and Mental Health, University of Cape Town, Cape Town, South Africa Full list of author information is available at the end of the article
}

development $[8,9]$ warrants further research to understand the disorder's aetiology, identify at-risk populations and develop effective preventive and therapeutic interventions.

The global evidence base on risk factors for perinatal depression is growing. Several factors have systematically been reported in low- and middle-income countries (LMICs) and high-income countries (HICs), such as a history of depression, social conflict, and lack of social support from family or partner [10-13]. Younger age, lower education status and being single are among the few demographic risk factors which have received some,

(c) The Author(s). 2019 Open Access This article is distributed under the terms of the Creative Commons Attribution 4.0 International License (http://creativecommons.org/licenses/by/4.0/), which permits unrestricted use, distribution, and 
but mixed evidence, for both antenatal and postnatal depression $[9,11-13]$. The evidence for socio-economic risk factors is mixed in South Africa [3, 4, 14]. However, food insecurity, defined as the inability to access a sufficient quantity of healthy food on a daily basis and reported by $38 \%$ of households in South Africa [15], has consistently been identified as a risk factor for antenatal and postnatal depression in the Western Cape [5, 14, 16, 17]. Intimate partner violence (IPV) has also been reported as a risk factor for perinatal depression in South Africa [3, 18-20], where IPV is common and is reported by more than $40 \%$ of pregnant women [21].

Fewer studies have focused on identifying healthrelated predictors of perinatal depression, yet these are particularly relevant in LMICs. In South Africa, the prevalence rate of women aged 15 to 49 living with HIV is approximately $24 \%$ [22], and evidence indicates that perinatal depression is more common among HIVpositive women [19]. The rates of alcohol consumption per capita are also very high in South Africa [23]: in recent studies conducted in low-income areas of Cape Town, hazardous drinking was reported by $7.1 \%$ of women at 8 months gestation [24], and by $16 \%$ of women three months after giving birth, indicating a level of alcohol consumption likely to have adverse health consequences [5]. Evidence suggests an association between alcohol use during pregnancy and postpartum depression [5, 24], though none has been found with antenatal depression [1].

The heterogeneity in risk factors identified for antenatal or postnatal depression highlight the complexity of this disorder's aetiology and course. The fact that most of the evidence is based on cross-sectional studies further limits our understanding of the factors associated with the onset, severity and chronicity of depressive symptoms during the perinatal period. Recent literature has used latent modelling techniques to investigate the heterogeneity of depression, both in terms of symptom profiles and trajectories [25]. Two systematic reviews have summarised the evidence using such modelling techniques in the context of perinatal depressive symptoms [26, 27]. Both reviews identified the most commonly reported trajectories to be a chronically severe and a chronically low symptom level trajectory. Transient trajectories were also reported, some of which suggested a natural remission among some women, despite similar severe levels of depressive symptoms antenatally compared to those suffering from chronic severe symptoms throughout the perinatal period [28-31]. Baron et al. [26] also point that predictors identified for several trajectories were not consistent across studies and did not distinguish women with chronic symptoms from those who presented transient trajectories.
Identifying such predictors would be especially useful in low-resource settings such as South Africa, since the use of screening instruments to identify women at risk of depression, without effective referral and treatment mechanisms, can overburden already weak and limited mental health services [32]. Indeed, being able to identify women who are most likely to suffer from chronic symptoms from those whose symptoms may abate naturally with minimal intervention may help streamline referrals and help target women who are most at risk. Unfortunately, as both reviews highlight, there is a dearth of evidence from LMICs. Only one LMIC study was conducted, among West African perinatal women [33]. The inclusion criteria meant, however, that the sample was a particularly low-risk group, and neither chronically severe or initially severe trajectories were identified. Given the gap in the literature, the aim of this study was to identify trajectories of perinatal depressive symptoms and their predictors among low-income South African women who were already at risk of depression during pregnancy.

\section{Methods \\ Design and setting}

This study is a secondary analysis of data collected for a randomised controlled trial (RCT) assessing the costeffectiveness of a brief psychosocial intervention for perinatal depression among 425 pregnant women at risk of depression living in Khayelitsha, a peri-urban informal settlement on the outskirts of Cape Town, South Africa. The poor living conditions, high crime rates and population density of Khayelitsha resembles that of the other informal settlements in South Africa [34-36]. The psychosocial intervention did not have an effect on women's depressive symptoms [37], which allowed the use of this sample for the purpose of the present study. The recruitment and data collection methods have been described previously [38], and are briefly reviewed here.

\section{Participants}

Recruitment took place in two community health centres in Khayelitsha. Pregnant women were screened for depressive symptoms during their first antenatal clinic booking, using the Edinburgh Postnatal Depression Scale (EPDS; [39]). The EPDS is a 10-item Likert-scale questionnaire assessing a range of depressive symptoms, such as anhedonia, somatic symptoms and suicide ideation. Its internal structure was acceptable among isiXhosaspeaking women in Khayelitsha [40]. Another validation study, conducted in an informal settlement in Johannesburg, suggests that a cut-off of 13 is optimal to indicate a risk for depression, with a sensitivity and specificity of 80 and $76.6 \%$, respectively [41]. Women who were at least 18 years of age, spoke isiXhosa, were in their first 
or second trimester and scored 13 or above on the EPDS were eligible for enrolment. For this study, participants of babies who had low birth weight $(<2.5 \mathrm{~kg} ; n=27)$ or who were premature $(<37$ weeks gestation; $n=43)$ were included in the analysis. However, participants who died, who experienced a miscarriage, or whose baby died during the course of the study were excluded from the analyses $(n=42)$. Besides greater levels of functioning among participants excluded from the analysis (median $=19.4$; interquartile range $(\mathrm{IQR})=8.3-30.6) \mathrm{com}$ pared to those included (median $=29.2 ; \mathrm{IQR}=16.7-41.7$; $\mathrm{U}=-2.64 ; p=0.008)-$, the baseline demographic, clinical or social characteristics of participants excluded from and included in the analysis did not differ [37].

\section{Procedure}

Once enrolled, participants were randomised into either a psychosocial intervention or enhanced usual care. The psychosocial intervention was provided by trained community health workers and consisted of six counselling sessions which included elements of psycho-education on depression and pregnancy, problem solving, behavioural activation and healthy thinking [42]. The enhanced usual care consisted of monthly phone calls for three months, where participants were asked a series of question relating to their health, suicide risk and recent life events. Phone calls lasted no more than five minutes, and were conducted by two separate community health workers, who were trained to conduct the phone calls, but were not trained in counselling. More details about the interventions and training are provided in Lund et al. [38]. All participants received the same regular antenatal care available at the clinics, which typically involves medical management of pregnancy, HIV testing and Prevention of Mother to Child Transmission care. An assessment was conducted at recruitment, and then again at eight months gestation, and three months and 12 months after giving birth. This was done by two fieldworkers who were blind to the participant's arm allocation.

\section{Measurements}

All assessments covered a range of mental health, health, social and economic measures. The baseline assessment also included socio-demographic questions [38]. Only age, education and marital status were considered potential demographic predictors and included in the analyses, as these characteristics are routinely collected during the first antenatal visits in South Africa.

\section{Health characteristics}

Depressive symptoms were primarily assessed using Potts et al. [43]'s 17-item version of the Hamilton Depression Rating Scale (HDRS) [44]. Scores range from
0 to 54 ; a higher score suggesting greater symptom severity. A cut-off of 17 has been suggested as indicating clinically significant depressive symptoms [45]. A more structured isiXhosa version of the HDRS was developed for the RCT for use by non-clinicians: this adapted version was validated and showed good construct validity and internal consistency (Cronbach's Alpha $=0.74$ ), and the inter-rater ( 0.97 to 0.98$)$ and test-retest reliability (0.90) were excellent [46].

The Mini International Neuropsychiatric Interview (MINI) 6.0 [47] Major Depressive Episode and Suicidality modules were used to assess current depression and suicidality risk, respectively. A lifetime diagnosis of depression was also assessed using the Major Depressive Episode module. The MINI 6.0 is a brief diagnostic interview which has been used as a gold standard in diverse populations, including among HIV-infected patients in South Africa $[48,49]$. High risk of suicide was defined as a score of 17 or more [50]. Participants who reported a high risk of suicide were immediately referred to see a psychiatric nurse, located in the same community health centre.

The World Health Organization (WHO) Disability Assessment Schedule (WHODAS 2.0; 12-item) [51] was used to determine the participants' level of impaired functioning. The item-response theory-based scoring was used, generating a score between 0 and 100, with greater scores suggesting greater impairment. The WHODAS 2.0 has good reliability and validity across cultures and population type [51]. As recommended by Schneider et al. [52], the WHODAS 2.0 was complemented with the Cape Town Functional Assessment Instrument (FAI), developed specifically for and validated among pregnant and postnatal women in this study and is meant to reflect more specific domains of functioning among this population [52]. It is a 10-item questionnaire, with responses ranging from "no difficulty" to "can never do the task". A "not applicable" option is also available, so total scores are calculated by dividing the sum of item scores by the number of items responded to. The total score ranges from 0 to 4 , with a greater score suggesting greater impairment.

Finally, alcohol use was assessed using the Alcohol Use Disorder Identification Test (AUDIT) [53], a 10item questionnaire, developed by the WHO, to identify alcohol misuse. Scores range from 0 to 40 , with greater scores indicating greater alcohol misuse. The AUDIT has been used to assess alcohol consumption habits in both men and women in the Cape Town region [54, 55]. The recommended cutoff for heavy or binge drinking among women in South Africa is 5, based on a validation study among a nationally representative sample [56]. HIV status was also recorded. 


\section{Social characteristics}

The Multidimensional Scale of Perceived Social Support (MSPSS) [57], a 12-item 7-point Likert Scale questionnaire, was used to assess perceived emotional support from family, friends and a 'special person'. Here, a 'special person' refers to a significant other, or a person with whom the participant has a close emotional relationship and is involved in their day-to-day lives. Overall scores range from 0 to 84, with higher scores suggesting greater perceived support. The scale has been validated in several LMICs [58, 59], including highschool students in Cape Town, South Africa $[60,61]$. Subscale scores were also calculated. IPV was assessed by asking participants if they had experienced physical (e.g. kicked, slapped, beaten) or sexual abuse by their partner in the past three months.

\section{Economic characteristics}

The Household Food Insecurity Access Scale (HFIAS) [62] is a 9-item questionnaire assessing three dimensions of food insecurity. It was previously used in a study among postnatal depressed women in Khayelitsha [5]. The HFIAS score was binarised so that participants were either considered severely food insecure or not, as done in previous research in other low-income settings [5, 63]. A proxy for socio-economic status was also developed using multiple correspondence analysis, where economic-related variables were analysed to create an asset-based score [64]. Variables included, but were not limited to, education, employment status, main source of income, whether the household income is fixed, housing characteristics and access to amenities. The score was transformed into a binary variable indicating whether participants were in the lower (below median asset score) or higher wealth category (at or above median asset score).

Instruments which had already been translated and validated in isiXhosa in previous studies (such as the EPDS, MINI, AUDIT, FAI and HFIAS) were reviewed by a translator for accuracy. All other sections in the assessments were translated into isiXhosa and back-translated to English.

\section{Analysis \\ Identification of trajectories}

The first stage of the analysis, conducted in Mplus version 8 [65], consisted of conducting growth mixture modelling (GMM), a method which combines growth curves with latent modelling. GMM allows investigators to explore groups of individuals with similar profile trajectories (classes) and allows for individual variability within latent classes. The HDRS scores at the four timepoints (recruitment, 8 months gestation, and 3 and 12 months postpartum) were used to create latent trajectories. This instrument, rather than the EPDS, was used as it more sensitive to change [66].

Scores on the HDRS did not differ significantly between the control and intervention arms at any assessment [37], so participants from the two arms were analysed together. However, attrition in the intervention arm (19.6\%) was higher than that in the control arm (6.5\%), though no differences were found in baseline characteristics between participants who were lost to follow-up and those who were followed-up. To account for differences in attrition, arm allocation was included as a covariate in all growth mixture models. Missing data were assumed to be missing at random and were dealt with using robust maximum likelihood estimation. To represent non-equidistant time points of assessments, factor loadings were fixed to $0,0.3,0.7$ and 1.6, to represent assessments at baseline, and then 3 months, 7 months and 16 months after baseline; time in months was divided by 10 to avoid non-convergence of the models [67]. An inspection of the individual data suggested heterogeneous, non-linear trends. Goodness of fit values generated from preliminary one-class (non-mixture) analyses indicated that a quadratic change function fitted the data best [68], so a quadratic pattern was introduced in subsequent models (see Additional file 1).

A series of mixture models were run, first assuming no variation within trajectory classes (intercepts and slope variance fixed at 0 - latent class growth analysis [LCGA]), and then allowing free estimates of means and variances for latent variables (GMM). Small and nonsignificant negative residual variances for HDRS scores at 12 months were dealt with by fixing the residual variance to a value close to 0 [67].

\section{Selection of optimal model}

Models with increasing number of classes were fitted against the data, and compared using standard statistical measures: the Bayesian Information Criterion (BIC) [69], the Akaike Information Criterion (AIC) [70] and entropy [71]. Priority was given to entropy in cases where fit indices between two models were relatively similar [72]. Given the relatively small sample size of the RCT, model solutions that included a class that comprised less than $5 \%$ of the sample were avoided. Average probability of class membership for each estimated class (posterior probability) were also used as a criterion for model fit. Successive models with different number of classes were compared using the Lo-Mendell-Rubin Test (LMRT) [73] and Bootstrap Likelihood Ratio Test (BLRT) [74]. Finally, the shape and theoretical interpretability of the trajectory classes were also taken into account. Once the optimal model was selected, participants were assigned to a latent trajectory class based on their highest posterior probability. 
To assess whether the psychosocial intervention had an effect on latent trajectories generated by the GMM, a sensitivity analysis was also conducted by running an unadjusted GMM, this time including the arm variable within the GMM model, so that trajectories would be generated per arm [75, 76].

\section{Identification of risk factors}

In the second stage of the analysis conducted in Stata 14, each demographic, health, social and economic variable collected at baseline was entered as a single predictor in an unadjusted logistic regression, with class membership as the outcome. Odds ratios (OR) with 95\% confidence intervals are reported, with a significance level set at 5\%. Univariate, rather than multivariate, analyses were preferable given that the objective of the study was to identify high-risk groups more likely to suffer from severe and chronic symptoms, rather than to understand the complex interactions of risk factors leading to chronic depressive symptoms.

\section{Results}

\section{Overview of sample}

The characteristics of the sample at baseline are presented in Table 1. On average, participants were 27 years of age (standard deviation $(\mathrm{SD})=5.56$ ). The majority of participants reported not finishing high school $(n=225,58.6 \%)$, not living with a partner $(n=$ $254,66.2 \%)$, and were not employed or still studying $(n=207,53.9 \%)$. Over a quarter reported being severely food insecure $(n=112,29.2 \%)$. A similar proportion reported being HIV-positive $(\mathrm{n}=112,30.1 \%)$ and drinking heavily during pregnancy $(n=114$, $29.7 \%)$. Over $40 \% \quad(n=157)$ were diagnosed with current depression on the MINI, $17.7 \%(n=68)$ were considered at risk for suicide, and $31.8 \%(n=122)$ had a history of depression. The mean number of assessments conducted was $3.5 ; 88.0 \%(n=338)$ of participants received at least three assessments, and 65.6\% $(n=252)$ received all four assessments.

\section{Identification of trajectories}

Table 2 provides the fit information criteria of the models generated through LCGA and GMM. Smaller values of BIC and AIC suggest a better model fit, while entropy values closest to 1 suggest better classification. Models generated through GMM fitted the data better than the LCGA models. Fit indices indicated that a 2-class model was optimal $(\mathrm{BIC}=$ 7797.269; $\quad$ AIC $=7722.207)$, with an entropy $(0.816)$ above the suggested minimum of 0.8 [77]. The 3-class model had a slightly lower AIC value (7708.730) and generated a small but interesting class characterised by a clinically different trend compared to the other two classes (see Additional file 2 for a graphical representation of the trajectories). However, the entropy was lower, and the model fit not significantly improved from the 2-class model according to the LMRT and BLRT. Taking these into consideration, and for reasons of parsimony, the 2class model was selected.

The mean HDRS scores of women allocated to the two classes are presented in Fig. 1. The antenatal only class is characterised by moderate levels of depressive symptoms at recruitment (mean $=15.0$, $\mathrm{SD}=4.28$ ), which decrease steadily over pregnancy and early postpartum, then stabilise by 12 months postpartum (mean $=9.3, \mathrm{SD}=3.82)$. The majority of the sample were allocated to this trajectory $(n=351$, 91.4\%). The antenatal and postnatal class represents a minority $(n=33,8.6 \%)$ with symptom levels above the recommended clinical cut-off of 17 at recruitment (mean $=22.1, \mathrm{SD}=4.67)$, which decline to moderate levels until 3-month postpartum (mean $=12.9, \mathrm{SD}=$ 5.40 ), but worsen again at 12-month postpartum to reach a mean of $19.3(\mathrm{SD}=3.49)$.

Results of the sensitivity analysis, not presented here, suggest that a 2-class model per arm was the most optimal. The trajectories and sample proportions generated for each arm were similar to those presented when both arms were combined.

\section{Predictors of trajectories}

The results of the unadjusted logistic regressions are presented in Table 3. The antenatal only class was used as the reference class. None of the baseline demographic variables differed between the two classes. Also, neither employment nor socio-economic status were significant predictors of class, however food insecurity was: the odds of being classified in the antenatal and postnatal trajectory were 2.5 times greater (95\% CI: 1.21, 5.15 ; $p=0.013)$ among participants who reported being severely food insecure.

Greater overall levels of social support at baseline decreased the odds of belonging to the antenatal and postnatal class $(\mathrm{OR}=0.97,95 \% \mathrm{CI}: 0.95,0.99 ; p=$ $0.011)$. However, only a greater level of family support $(\mathrm{OR}=0.91,95 \% \mathrm{CI}: 0.86,0.96 ; p=0.001)$ or greater level of support from a significant other $(\mathrm{OR}=0.94$, 95\% CI: $0.88,1.00 ; p=0.046)$ decreased the odds of being classified in the antenatal and postnatal class. Those who reported experiencing IPV at baseline were also 2.8 times more likely (95\% CI: 1.23, 6.52; $p=0.014)$ to belong to the antenatal and postnatal class.

Besides a HIV-positive status, all other healthrelated characteristics at baseline were associated with class membership: the odds of belonging to the antenatal and postnatal class were greater among 
Table 1 Characteristics of the sample, by class

\begin{tabular}{|c|c|c|c|c|c|c|c|c|c|}
\hline \multirow[t]{2}{*}{ Variable } & \multicolumn{3}{|c|}{ Total $(N=384)$} & \multicolumn{3}{|c|}{ Antenatal only $(N=351)$} & \multicolumn{3}{|c|}{ Antenatal \& postnatal $(N=33)$} \\
\hline & $\mathrm{n}$ & $\%$ & Mean (SD) & $\mathrm{n}$ & $\%$ & Mean (SD) & $n$ & $\%$ & Mean (SD) \\
\hline \multicolumn{10}{|l|}{ Demographic characteristics } \\
\hline Age & & & $27.2(5.61)$ & & & $27.0(5.54)$ & & & $28.7(6.23)$ \\
\hline Gestation & & & $17.2(5.71)$ & & & $17.1(5.74)$ & & & $18.2(5.31)$ \\
\hline Did not complete high school & 225 & 58.6 & & 203 & 57.8 & & 22 & 66.7 & \\
\hline VDoesn't live with partner & 254 & 66.2 & & 232 & 66.1 & & 22 & 66.7 & \\
\hline \multicolumn{10}{|l|}{ Economic measures } \\
\hline Unemployed/studying & 207 & 53.9 & & 190 & 54.1 & & 17 & 51.5 & \\
\hline Lower wealth (socio-economic status) & 195 & 50.8 & & 176 & 50.1 & & 19 & 57.6 & \\
\hline Severely food insecure & 112 & 29.2 & & 96 & 27.4 & & 16 & 48.5 & \\
\hline \multicolumn{10}{|l|}{ Social characteristics } \\
\hline Intimate partner violence (past 3 months) & 50 & 13.0 & & 41 & 11.7 & & 9 & 27.3 & \\
\hline Overall social support & & & $58.3(12.91)$ & & & $58.8(12.56)$ & & & $52.8(15.3)$ \\
\hline Social support from family & & & $20.1(5.70)$ & & & $20.4(5.48)$ & & & $16.9(6.97)$ \\
\hline Social support from friends & & & $16.0(6.24)$ & & & $16.0(6.19)$ & & & $15.3(6.77)$ \\
\hline Social support from special person & & & $22.2(4.75)$ & & & $22.4(4.57)$ & & & $20.6(6.18)$ \\
\hline \multicolumn{10}{|l|}{ Health characteristics } \\
\hline Functioning (WHODAS) & & & $29.8(17.64)$ & & & $28.9(17.13)$ & & & $39.3(20.34)$ \\
\hline Functioning (FAl) & & & $0.8(0.5)$ & & & $0.8(0.5)$ & & & $1.2(0.6)$ \\
\hline Heavy drinking & 114 & 29.7 & & 99 & 28.2 & & 15 & 45.5 & \\
\hline HIV positive status & 112 & 30.1 & & 100 & 29.3 & & 12 & 38.7 & \\
\hline Current diagnosis of depression (MINI) & 157 & 40.9 & & 136 & 38.8 & & 21 & 63.6 & \\
\hline Lifetime diagnosis of depression (MINI) & 122 & 31.8 & & 104 & 29.6 & & 18 & 54.6 & \\
\hline High suicide risk (MINI) & 68 & 17.7 & & 57 & 16.2 & & 11 & 33.3 & \\
\hline
\end{tabular}

$S D=$ standard deviation

participants reporting greater functional impairment, 2.12, 95\% CI: 1.03, 4.37; $p=0.042)$, had a current $(\mathrm{OR}=$ measured with the WHODAS (OR $=1.03,95 \%$ CI: $1.02, \quad 2.77,95 \%$ CI: $1.32,5.80 ; p=0.007$ ) or lifetime diagnosis of $1.06 ; p=0.002)$ and FAI (OR $=5.74,95 \% \mathrm{CI}: 2.82,11.70 ; \quad$ depression $(\mathrm{OR}=2.85,95 \% \mathrm{CI}: 1.38,5.87 ; p=0.004)$, and $p<0.001)$; the odds were also greater among participants were at high risk of suicide $(\mathrm{OR}=2.58,95 \% \mathrm{CI}: 1.19,5.61$; who reported heavy drinking during pregnancy $(\mathrm{OR}=p=0.017)$.

Table 2 Latent class growth analysis and growth mixture modelling: comparisons of models

\begin{tabular}{|c|c|c|c|c|c|c|}
\hline Classes & $\mathrm{BIC}$ & $\mathrm{AIC}$ & Entropy & Size (\%) of smallest class & LMRT statistic ( $p$-value) & BLRT statistic ( $p$-value) \\
\hline \multicolumn{7}{|c|}{ Quadratic LCGA ${ }^{a}$} \\
\hline 2 & 7825.996 & 7770.687 & 0.742 & 26.0 & $203.838(<0.001)$ & $-3977.544(<0.001)$ \\
\hline 3 & 7809.547 & 7738.435 & 0.750 & 4.6 & $38.630(0.034)$ & $-3871.344(0.030)$ \\
\hline 4 & 7817.215 & 7730.301 & 0.718 & 4.6 & $15.483(0.304)$ & $-3851.218(0.288)$ \\
\hline 5 & 7828.413 & 7725.696 & 0.584 & 3.9 & $12.097(0.657)$ & $-3843.151(0.647)$ \\
\hline \multicolumn{7}{|c|}{ Quadratic GMM ${ }^{\text {b }}$} \\
\hline 2 & 7797.269 & 7722.207 & 0.816 & 8.6 & $38.789(<0.001)$ & $-3862.313(<0.001)$ \\
\hline 3 & 7799.594 & 7708.730 & 0.807 & 4.2 & $20.612(0.503)$ & $-3842.104(0.488)$ \\
\hline 4 & 7814.876 & 7708.209 & 0.762 & 1.6 & $8.177(0.234)$ & $-3831.365(0.229)$ \\
\hline 5 & 7822.705 & 7700.235 & 0.771 & 1.3 & $15.330(0.123)$ & -3827.105 (0.115) \\
\hline
\end{tabular}

$A I C=$ Akaike Information Criterion, $B I C=$ Bayesian Information Criterion; BLRT= Bootstrap Likelihood Ratio Test, $L M R T=$ Lo-Mendell-Rubin Test; ${ }^{\mathrm{a}}$ Latent curve growth analysis; ${ }^{b}$ growth mixture modelling 


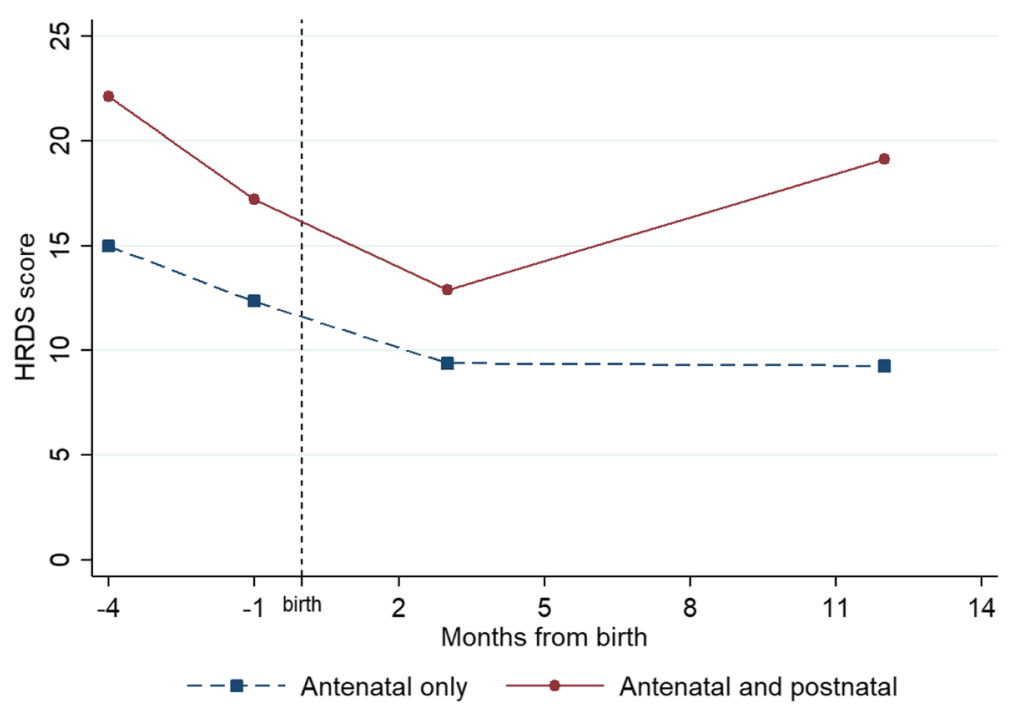

Fig. 1 Mean HDRS curves of the GMM 2-class solution; legend: Note: Missing data for antenatal only trajectory: 8 months gestation ( $n=84,23.9 \%)$, 3 months postpartum $(n=43,12.3 \%)$ and 12 months postpartum $(n=61,17.4 \%)$; missing data for antenatal and postnatal trajectory: 8 months gestation ( $n=10,30.3 \%), 3$ months postpartum $(n=4,12.1 \%)$ and 12 months postpartum $(n=3,9.1 \%)$

\section{Discussion}

The aim of this study was to identify different trajectories of perinatal depressive symptoms and their predictors among low-income South African women at risk of depression antenatally. Through GMM, we were able to identify two subgroups of women with different severity and chronicity of perinatal depressive symptoms: an antenatal only trajectory and an antenatal and postnatal trajectory. On the one hand, the antenatal only trajectory is consistent with previous longitudinal studies reporting a natural remission group [28-31]. This means that, without intervention, and despite initially mild to moderate depressive symptoms during the first or second trimester, the majority of women showed improvements in their symptoms throughout the remainder of the perinatal period. The antenatal and postnatal trajectory, on the other hand, suggests that there was a minority of women who did not see their symptoms remit naturally and remained at risk of depression for most of the perinatal period. So, an initial decline in symptom severity throughout pregnancy and first three months postpartum was observed for both trajectories. This suggests that screening within the first weeks after birth to identify women at risk of postnatal depression may not be effective in identifying at-risk women later in the postpartum period.

The findings also suggest that women who are at risk of chronic depressive symptoms can be differentiated from those showing a natural remission on a range of psychosocial and health-related characteristics during pregnancy, other than their initial depressive symptom severity. Women were more likely to belong to the antenatal and postnatal class when they reported being severely food insecure, experienced physical or sexual IPV, had lower support from family or significant other and reported problematic drinking during pregnancy. They were also at greater risk of committing suicide and were more likely to have a current or lifetime diagnosis of depression. These findings support previous evidence of the association between perinatal depressive symptoms in South African women and suicide risk [78], hazardous drinking $[5,24]$, IPV $[3,19,20]$ and food insecurity $[5,14,16,17]$. It is interesting to note that the same association between suicidal risk and trajectories were found when the suicide item was excluded from the HDRS scores used for the GMM and post-hoc analyses (results not presented here). Thus, the association found between suicidal risk and trajectories was not confounded by the inclusion of the suicide item in the HDRS.

Women who were more likely to suffer from chronic depressive symptoms therefore seem to have presented with a higher risk profile during pregnancy in terms of social, economic, health and mental health characteristics. This has important implications on referral and treatment procedures in settings where there are limited mental health resources. First, given the greater likelihood of women diagnosed with depression to be allocated to the 'antenatal and postnatal' trajectory, our findings indicate how effective a diagnosis would be in detecting women at higher risk for chronic symptoms. Indeed, some researchers have suggested that conducting a formal diagnostic assessment by a mental health professional could be an efficient use of resources if 
Table 3 Unadjusted logistic regression, with antenatal only class as reference

\begin{tabular}{|c|c|c|c|}
\hline \multirow[b]{2}{*}{ Variable } & \multicolumn{3}{|c|}{ Antenatal \& Postnatal $(n=33)$} \\
\hline & $\overline{\mathrm{OR}}$ & $95 \% \mathrm{Cl}$ & $p$ value \\
\hline \multicolumn{4}{|l|}{ Baseline demographic characteristics } \\
\hline Age & 1.05 & $0.99-1.12$ & 0.112 \\
\hline \multicolumn{4}{|l|}{ Education level } \\
\hline Grade $0-11$ & ref & - & \\
\hline Grade 12 or more & 0.69 & $0.32-1.46$ & 0.327 \\
\hline \multicolumn{4}{|l|}{ Marital status } \\
\hline Lives with partner & ref & & \\
\hline Doesn't live with partner & 1.03 & $0.48-2.19$ & 0.947 \\
\hline \multicolumn{4}{|l|}{ Baseline economic measures } \\
\hline \multicolumn{4}{|l|}{ Employment } \\
\hline Employed & ref & & \\
\hline Unemployed/studying & 0.90 & $0.44-1.84$ & 0.773 \\
\hline \multicolumn{4}{|l|}{ Socio-economic status } \\
\hline Lower wealth & ref & & \\
\hline Higher wealth & 0.74 & $0.36-1.52$ & 0.415 \\
\hline \multicolumn{4}{|l|}{ Food status } \\
\hline Not severely food insecure & ref & & \\
\hline Severely food insecure & 2.50 & $1.21-5.15$ & 0.013 \\
\hline \multicolumn{4}{|l|}{ Baseline social characteristics } \\
\hline Intimate partner violence (IPV) & 2.84 & $1.23-6.52$ & 0.014 \\
\hline Overall social support & 0.97 & $0.95-0.99$ & 0.011 \\
\hline Social support from family & 0.91 & $0.86-0.96$ & 0.001 \\
\hline Social support from friends & 0.98 & $0.93-1.04$ & 0.498 \\
\hline Social support from significant other & 0.94 & $0.88-1.00$ & 0.046 \\
\hline \multicolumn{4}{|l|}{ Baseline health characteristics } \\
\hline Functioning (WHODAS) & 1.03 & $1.02-1.06$ & 0.002 \\
\hline Functioning (FAl score) & 5.74 & $2.82-11.70$ & $<0.001$ \\
\hline Heavy drinking ${ }^{b}$ & 2.12 & $1.03-4.37$ & 0.042 \\
\hline HIV positive status $^{c}$ & 1.52 & $0.71-3.25$ & 0.278 \\
\hline Current diagnosis of depression (MINI) ${ }^{d}$ & 2.77 & $1.32-5.80$ & 0.007 \\
\hline 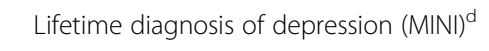 & 2.85 & $1.38-5.87$ & 0.004 \\
\hline High suicide risk (MINI) ${ }^{d}$ & 2.58 & $1.19-5.61$ & 0.017 \\
\hline
\end{tabular}

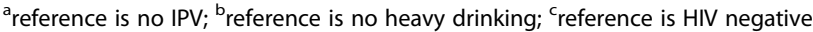
status; ${ }^{d}$ reference is absence of diagnosis/risk; OR= odds ratio; $95 \% \mathrm{Cl}=95 \%$ confidence interval

done in a stepped care manner, that is, if conducted only among women who screened positive on a screening instrument $[79,80]$. Alternatively, food insecurity, alcohol use during pregnancy and social support are factors which are relatively easy to assess during pregnancy; only referring to care women with severe symptoms but who also present with greater functional impairment, or who report alcohol use or low support during pregnancy, would limit referrals made and allow the limited mental health services to target women who are at most risk. Women who report severe depressive symptoms but who do not present such risk factors could instead be referred to peer support groups or community-based care.

It is important to note that, despite a decrease in symptoms among the antenatal only trajectory, symptom levels remained within the mild range, albeit at the lower end [81], throughout the postpartum period. Yet studies have shown that chronic symptoms, even if mild or moderate, can have adverse effects on child outcomes [27]. It was beyond the scope of this study to assess whether women in different trajectory groups reported different child and maternal outcomes, however future studies should consider investigating the long-term effects of chronic trajectories on mother and child health in LMICs, across all levels of severity.

It is difficult to compare the present findings to those reported by Barthel et al. [33]'s, the only other study using growth curve mixture models (GCMM) to assess perinatal depressive symptoms among women living in two LMICs. In their study of West African perinatal women, the authors are likely to have excluded a highrisk group by excluding women whose children were born prematurely or had a low birth weight from their study. This is supported by the fact that a chronically severe trajectory was not identified in their study, despite this trajectory being commonly reported in most studies using GCMM [26]. Instead, three trajectories were reported: a chronically low-symptom trajectory, and two transient trajectories, characterised by severe symptoms either early or late in the postpartum period, before returning to low levels [33]. This supports previous studies, which have reported trajectories with initially low levels of depressive symptoms early in pregnancy, which increase later in the perinatal period $[30,82]$. It is therefore vital that future studies include women with a range of symptom levels at recruitment, to ensure that all potential trajectories and associated predictors be identified.

\section{Limitations}

The study provides useful evidence on the different trajectories of depressive symptoms of women at risk of depression at their first antenatal visit. However, several limitations need to be highlighted. First, the level of uncertainly associated with the predicted trajectory membership was not controlled for in the post-hoc analyses, which instead treated trajectories as an observed variable. The entropy was above the usual cutoff of 0.80 , however, suggesting adequate classification of participants and considered sufficient to conduct posthoc regression analyses [77]. Second, with a greater sample size, the three-class model identified through 
GMM may have been more optimal and more representative of the course of symptoms among low-income high-risk women in this setting.

Third, it was established that the reduction in symptoms among the antenatal and postnatal trajectory was unlikely to be due to the psychosocial intervention assessed through the RCT. Indeed, there was no difference in HDRS scores at any point between the control and treatment arms, and the allocation arm was controlled for in the growth mixture model. The sensitivity analysis also revealed that the optimal model generated through GMM was similar when trajectories were generated per allocation arm. However, it is possible that the trajectories identified in this study may partly reflect the combined effect of the enhanced usual care and psychosocial interventions. Indeed, it has previously been suggested that the lack of difference between control and intervention arms, a common phenomenon of RCTs assessing behavioural interventions, may be due to the enhanced usual care having an effect on participants in the control arm, rather than a lack of effect of the intervention itself [83]. Further studies should therefore run the same analyses among women at risk of depression but who did not receive any intervention. A final limitation is the fact that anxiety was not assessed. Anxiety and depression are often comorbid during the perinatal period [13], and the prevalence of anxiety among South African perinatal population is sometimes even greater than that of postnatal depression [4]. Future research should therefore investigate whether comorbid anxiety symptoms predict a different course of perinatal depressive symptoms over the perinatal period.

\section{Conclusion}

This study is one of first studies to investigate the severity and course of depressive symptoms during the perinatal period using GMM in a LMIC. Despite the limitations highlighted above, the findings indicate that perinatal women at risk of depression antenatally cannot be considered as a uniform group. The findings highlight the importance of moving beyond a symptom-based identification of mental illness and towards a screening procedure that takes into account the importance of psychosocial determinants in the development and the course of perinatal depressive symptoms. By doing so, referral systems, as well as timing and target populations for interventions addressing perinatal depressive symptoms can be established in a more efficient way, given the limited mental health resources in low-income South African settings and in other LMICs.

\section{Additional files}

Additional file 1: Results of the preliminary one-class (non-mixture) analyses. Description: Table summarising the statistics generated from one-class analyses - not essential for the main body of the text but may be useful to some readers. (DOCX $12 \mathrm{~kb}$ )

Additional file 2: Mean HDRS curves for the alternative 3-class growth mixture model. Description: Figure illustrating the mean HDRS score of participants had they been allocated to three trajectories identified in the alternative 3-class solution of the growth mixture model. (DOCX 69 kb)

\section{Abbreviations}

AIC: Akaike Information Criterion; AUDIT: Alcohol Use Disorder Identification Test; BIC: Bayesian Information Criterion; BLRT: Bootstrap Likelihood Ratio Test; EPDS: Edinburgh Postnatal Depression Scale; FAl: Functional Assessment Instrument; GCMM: Growth curve mixture model; GMM: Growth mixture modelling; HDRS: Hamilton Depression Rating Scale; HFIAS: Household Food Insecurity Access Scale; HICs: High-income countries; IPV: Intimate partner violence; IQR: Interquartile range; LCGA: Latent class growth analysis;

LMICs: Low- and middle-income countries; LMRT: Lo-Mendell Rubin Test; MINI: Mini International Neuropsychiatric Interview; MSPSS: Multidimensional Scale of Perceived Social Support; RCT: Randomised controlled trial; WHODAS: WHO Disability Assessment Schedule

\section{Acknowledgements}

This study was conducted as part of the Africa Focus on Intervention Research for Mental Health (AFFIRM) NIMH Collaborative research hub. The authors would like to acknowledge the statistical support provided by Dr. Annibale Cois, and would like to thank the fieldworkers and counsellors for their work during the course of the study.

\section{Authors' contributions'}

ECG analysed and interpreted the data, and was the lead contributor in writing the manuscript. MS and CL oversaw data collection and contributed to writing the manuscript. All authors read and approved the final manuscript.

\section{Funding}

The original RCT was supported by the National Institute of Mental Health of the National Institutes of Health [U19MH095699]. This study is an output of the PRogramme for Improving Mental health carE (PRIME), supported by the UK Department for International Development [201446]. The funders did not have any role in the study design, data collection, analysis or interpretation of the data, or in the writing of the manuscript.

\section{Availability of data and materials}

The dataset used and analysed during the current study are not yet made available to the public, but are available from the corresponding author on reasonable request.

\section{Ethics approval and consent to participate}

The present study received ethical approval from the University of Cape Town Human Research Ethics Committee (HREC) (REF 835/2015), as did the randomised controlled trial on which this analysis is based (HREC REF $226 / 2011$ ). All participants recruited in this study provided informed written consent.

Consent for publication

Not applicable.

\section{Competing interests}

The authors declare that they have no competing interests.

\section{Author details}

${ }^{1}$ Alan J Flisher Centre for Public Mental Health, Department of Psychiatry and Mental Health, University of Cape Town, Cape Town, South Africa. ${ }^{2}$ Centre for Global Mental Health, Health Service and Population Research Department, Institute of Psychiatry, Psychology and Neuroscience, King's College London, London, UK. 
Received: 11 February 2019 Accepted: 9 June 2019

Published online: 14 June 2019

\section{References}

1. Hartley M, Tomlinson M, Greco E, Comulada WS, Stewart J, Le Roux I, et al Depressed mood in pregnancy: prevalence and correlates in two Cape Town peri-urban settlements. Reprod Health. 2011:8(9):4755-8.

2. Manikkam L, Burns JK. Antenatal depression and its risk factors: an urban prevalence study in KwaZulu-Natal. SAMJ: South African Medical Journal. 2012;102(12):940-4.

3. Brittain K, Myer L, Koen N, Koopowitz S, Donald KA, Barnett W, et al. Risk factors for antenatal depression and associations with infant birth outcomes: results from a south African birth cohort study. Paediatr Perinat Epidemiol. 2015;29(6):505-14.

4. Redinger $S$, Norris $S$, Pearson R, Richter $L$, Rochat T. First trimester antenatal depression and anxiety: prevalence and associated factors in an urban population in Soweto, South Africa. J Dev Orig Health Dis. 2017;9(1):30-40.

5. Dewing S, Tomlinson M, le Roux IM, Chopra M, Tsai AC. Food insecurity and its association with co-occurring postnatal depression, hazardous drinking, and suicidality among women in peri-urban South Africa. J Affect Disord. 2013;150(2):460-5

6. Verkuijl NE, Richter L, Norris SA, Stein A, Avan B, Ramchandani PG. Postnatal depressive symptoms and child psychological development at 10 years: a prospective study of longitudinal data from the south African birth to twenty cohort. Lancet Psychiatry. 2014;1(6):454-60.

7. Ramchandani PG, Richter LM, Stein A, Norris SA. Predictors of postnatal depression in an urban south African cohort. J Affect Disord. 2009;113(3):279-84.

8. Wachs TD, Black MM, Engle PL. Maternal depression: a global threat to children's health, development, and behavior and to human rights. Child Dev Perspect. 2009;3(1):51-9.

9. Gelaye B, Rondon MB, Araya R, Williams MA. Epidemiology of maternal depression, risk factors, and child outcomes in low-income and middleincome countries. Lancet Psychiatry. 2016;3(10):973-82.

10. Lancaster CA, Gold KJ, Flynn HA, Yoo H, Marcus SM, Davis MM. Risk factors for depressive symptoms during pregnancy: a systematic review. Am J Obstet Gynecol. 2010;202(1):5-14.

11. Sawyer A, Ayers S, Smith H. Pre-and postnatal psychological wellbeing in Africa: a systematic review. J Affect Disord. 2010;123(1):17-29.

12. Fisher J, MCd M, Patel V, Rahman A, Tran T, Holton S, et al. Prevalence and determinants of common perinatal mental disorders in women in low-and lower-middle-income countries: a systematic review. Bull World Health Organ. 2012;90(2):139-49.

13. Biaggi A, Conroy S, Pawlby S, Pariante CM. Identifying the women at risk of antenatal anxiety and depression: a systematic review. J Affect Disord. 2016;191:62-77.

14. van Heyningen $T$, Myer $L$, Onah M, Tomlinson M, Field S, Honikman S. Antenatal depression and adversity in urban South Africa. J Affect Disord. 2016;203:121-9.

15. Sorsdahl K, Slopen N, Siefert K, Seedat S, Stein DJ, Williams DR. Household food insufficiency and mental health in South Africa. J Epidemiol Community Health. 2011;65(5):426-31.

16. Tsai AC, Bangsberg DR, Frongillo EA, Hunt PW, Muzoora C, Martin JN, et al. Food insecurity, depression and the modifying role of social support among people living with HIV/AIDS in rural Uganda. Soc Sci Med. 2012;74(12):2012-9.

17. Abrahams Z, Lund C, Field S, Honikman S. Factors associated with household food insecurity and depression in pregnant south African women from a low socio-economic setting: a cross-sectional study. Soc Psychiatry Psychiatr Epidemiol. 2018;53(4):363-72.

18. Groves AK, Moodley D, McNaughton-Reyes L, Martin SL, Foshee V, Maman S. Prevalence, rates and correlates of intimate partner violence among south African women during pregnancy and the postpartum period. Matern Child Health J. 2015;19(3):487-95.

19. Peltzer K, Rodriguez VJ, Jones D. Prevalence of prenatal depression and associated factors among HIV-positive women in primary care in Mpumalanga province, South Africa. SAHARA-J. 2016;13(1):60-7.

20. Schneider M, Baron E, Davies T, Munodawafa M, Lund C. Patterns of intimate partner violence among perinatal women with depression symptoms in Khayelitsha. South Africa: a longitudinal analysis Global Mental Health. 2018;5:e13.
21. Sowa NA, Cholera R, Pence BW, Gaynes BN. Perinatal depression in HIV-infected African women: a systematic review. J Clin Psychiatry. 2015;76(10):1385-96

22. UNAIDS. UNAIDS Data 2017. Geneva, Switzerland: UNAIDS; 2017.

23. World Health Organization. Global status report on alcohol and health, 2014. Geneva, Switzerland: World Health Organization. Management of Substance Abuse Unit; 2014

24. Davis EC, Rotheram-Borus MJ, Weichle TW, Rezai R, Tomlinson M. Patterns of alcohol abuse, depression, and intimate partner violence among township mothers in South Africa over 5 years. AIDS Behav. 2017;21(2):174-82.

25. Nandi A, Beard J, Galea S. Epidemiologic heterogeneity of common mood and anxiety disorders over the lifecourse in the general population: a systematic review. BMC Psychiatry. 2009;9(1):31.

26. Baron E, Bass J, Murray SM, Schneider M, Lund C. A systematic review of growth curve mixture modelling literature investigating trajectories of perinatal depressive symptoms and associated risk factors. J Affect Disord. 2017:223:194-208.

27. Santos H, Tan X, Salomon R. Heterogeneity in perinatal depression: how far have we come? A systematic review. Arch Womens Ment Health. 2017;20(1):1-13

28. Christensen AL, Stuart EA, Perry DF, Le HN. Unintended pregnancy and perinatal depression trajectories in low-income, high-risk Hispanic immigrants. Prev Sci. 2011;12(3):289-99.

29. Lee CT, Stroo M, Fuemmeler B, Malhotra R, Østbye T. Trajectories of depressive symptoms over 2 years postpartum among overweight or obese women. Womens Health Issues. 2014;24(5):559-66.

30. Mora PA, Bennett IM, Elo IT, Mathew L, Coyne JC, Culhane JF. Distinct trajectories of perinatal depressive symptomatology: evidence from growth mixture modeling. Am J Epidemiol. 2009;169(1):24-32.

31. Vanska M, Punamaki R-L, Tolvanen A, Lindblom J, Flykt M, Unkila-Kallio $L$, et al. Maternal pre- and postnatal mental health trajectories and child mental health and development: prospective study in a normative and formerly infertile sample. Int J Behav Dev. 2011;35(6):517-31.

32. Kagee A, Tsai AC, Lund C, Tomlinson M. Screening for common mental disorders in low resource settings: reasons for caution and a way forward. Int Health. 2013:5(1):11-4.

33. Barthel D, Kriston L, Fordjour D, Mohammed Y, Kra-Yao ED, Kotchi CEB, et al. Trajectories of maternal ante-and postpartum depressive symptoms and their association with child-and mother-related characteristics in a west African birth cohort study. PLoS One. 2017:12(11):e0187267.

34. Statistics South Africa. Census 2011: Khayelitsha 2011 [updated 05 December 2018. Available from: http://www.statssa.gov.za/?page_id= 4286\&id=328.

35. Statistics South Africa. Census 2011: Mfuleni: Statistics South Africa; 2011 [Available from: http://www.statssa.gov.za/?page_id=4286\&id=324.

36. Statistics South Africa. Crime Stats: Khayelitsha: Statistics South Africa; 2018 [Available from: https://www.crimestatssa.com/precinct.php?id=1074.

37. Lund C, Schneider M, Baron E, Davies T, Munodawafa M, Honikman S, et al. Task-sharing of psychological treatment for antepartum depression in Khayelitsha, South Africa: Effects on antepartum and postpartum outcomes in an individual randomised controlled trial. Behav Res Ther. Under review; Under review.

38. Lund C, Schneider M, Davies T, Nyatsanza M, Honikman S, Bhana A, et al. Task sharing of a psychological intervention for maternal depression in Khayelitsha. South Africa: study protocol for a randomized controlled trial Trials. 2014;15:457.

39. Cox JL, Holden JM, Sagovsky R. Detection of postnatal depression. Development of the 10-item Edinburgh postnatal depression scale. Br J Psychiatry. 1987;150(6):782-6.

40. De Bruin GP, Swartz L, Tomlinson M, Cooper PJ, Molteno C. The factor structure of the Edinburgh postnatal depression scale in a south African peri-urban settlement. S Afr J Psychol. 2004;34(1):113-21.

41. Lawrie T, Hofmeyr G, De Jager M, Berk M. Validation of the Edinburgh postnatal depression scale on a cohort of south African women. S Afr Med J. 1998;88(10):1340-4.

42. Nyatsanza M, Schneider M, Davies T, Lund C. Filling the treatment gap: developing a task sharing counselling intervention for perinatal depression in Khayelitsha. South Africa BMC Psychiatry. 2016:16(1):164

43. Potts MK, Daniels M, Burnam MA, Wells KB. A structured interview version of the Hamilton depression rating scale: evidence of reliability and versatility of administration. J Psychiatr Res. 1990;24(4):335-50. 
44. Hamilton M. A rating scale for depression. J Neurol Neurosurg Psychiatry. 1960;23(1):56-62.

45. Zimmerman M, Martinez JH, Young D, Chelminski I, Dalrymple K. Severity classification on the Hamilton depression rating scale. J Affect Disord. 2013;150(2):384-8.

46. Davies T, Baron EC, Lund C, Schneider M. Adaptation and validation of a structured version of the Hamilton depression rating scale for use by nonclinicians in South Africa (AFFIRM-HDRS). International journal of mental health systems. Under review.

47. Sheehan DV, Lecrubier $Y$, Sheehan KH, Amorim P, Janavs J, Weiller E, et al. The MINI-international neuropsychiatric interview (MINI): the development and validation of a structured diagnostic psychiatric interview for DSM-IV and ICD-10. J Clin Psychiatry. 1998;59:22-33.

48. Lozano R, Naghavi M, Foreman K, Lim S, Shibuya K, Aboyans V, et al. Global and regional mortality from 235 causes of death for 20 age groups in 1990 and 2010: a systematic analysis for the global burden of disease study 2010. Lancet. 2013;380(9859):2095-128.

49. Murray CJ, Ezzati M, Flaxman AD, Lim S, Lozano R, Michaud C, et al. GBD 2010: a multi-investigator collaboration for global comparative descriptive epidemiology. Lancet. 2013;380(9859):2055-8.

50. Sheehan D, Lecrubier Y. MINI International Neuropsychiatric Interview English Version 6.0.0. [online]; 2010.

51. Üstün TB. Measuring health and disability: manual for WHO disability assessment schedule WHODAS 2.0. Geneva, Switzerland: World Health Organization; 2010.

52. Schneider M, Baron EC, Davies T, Bass J, Lund C. Making assessment locally relevant: measuring functioning for maternal depression in Khayelitsha. Cape Town Soc Psychiatry Psychiatr Epidemiol. 2015;50(5):797-806.

53. Saunders JB, Aasland OG, Babor TF, De la Fuente JR, Grant M. Development of the alcohol use disorders identification test (AUDIT). WHO collaborative project on early detection of persons with harmful alcohol consumption-II. Addiction. 1993;88(6):791-804.

54. Kalichman SC, Simbayi LC, Kaufman M, Cain D, Jooste S. Alcohol use and sexual risks for HIV/AIDS in sub-Saharan Africa: systematic review of empirical findings. Prev Sci. 2007;8(2):141-51

55. Kalichman SC, Simbayi LC, Vermaak R, Cain D, Smith G, Mthebu J, et al. Randomized trial of a community-based alcohol-related HIV risk-reduction intervention for men and women in Cape Town South Africa. Ann Behav Med. 2008;36(3):270-9.

56. Aalto M, Alho H, Halme JT, Seppä K. AUDIT and its abbreviated versions in detecting heavy and binge drinking in a general population survey. Drug Alcohol Depend. 2009;103:25-9.

57. Zimet GD, Dahlem NW, Zimet SG, Farley GK. The multidimensional scale of perceived social support. J Pers Assess. 1988;52(1):30-41.

58. Eker D, Arkar H. Perceived social support: psychometric properties of the MSPSS in normal and pathological groups in a developing country. Soc Psychiatry Psychiatr Epidemiol. 1995;30(3):121-6.

59. Doku PN, Dotse JE, Mensah KA. Perceived social support disparities among children affected by HIV/AIDS in Ghana: a cross-sectional survey. BMC Public Health. 2015;15(1):538.

60. Bruwer B, Emsley R, Kidd M, Lochner C, Seedat S. Psychometric properties of the multidimensional scale of perceived social support in youth Compr Psychiatry. 2008;49(2):195-201.

61. Rothon C, Stansfeld SA, Mathews C, Kleinhans A, Clark C, Lund C, et al. Reliability of self report questionnaires for epidemiological investigations of adolescent mental health in Cape Town, South Africa. J Child Adolesc Ment Health. 2011;23(2):119-28.

62. Coates J, Swindale A, Bilinski P. Household food insecurity access scale (HFIAS) for measurement of food access: Indicator guide. Washington DC: Fanta III; 2006.

63. Maxwell D, Vaitla B, Coates J. How do indicators of household food insecurity measure up? An empirical comparison from Ethiopia. Food Policy. 2014:47:107-16.

64. Booysen F, Van Der Berg S, Burger R, Von Maltitz M, Du Rand G. Using an asset index to assess trends in poverty in seven Sub-Saharan African countries. World Development. 2008;36(6):1113-30.

65. Muthén L, Muthén B. Mplus User's Guide. Los Angeles, CA: Muthén \& Muthén; 1998-2015.
66. Ballesteros J, Bobes J, Bulbena A, Luque A, Dal-Ré R, Ibarra N, et al. Sensitivity to change, discriminative performance, and cutoff criteria to define remission for embedded short scales of the Hamilton depression rating scale (HAMD). J Affect Disord. 2007;102(1-3):93-9.

67. Berlin KS, Parra GR, Williams NA. An introduction to latent variable mixture modeling (part 2): longitudinal latent class growth analysis and growth mixture models. J Pediatr Psychol. 2014;39(2):188-203.

68. Hu L, Bentler PM. Cutoff criteria for fit indexes in covariance structure analysis: conventional criteria versus new alternatives. Struct Equ Model Multidiscip J. 1999;6(1):1-55.

69. Raftery AE. Bayesian model selection in social research. Sociol Methodol. 1995:25:111-64.

70. Akaike H. Information measures and model selection. Bulletin of the International Statistical Institute. 1983;50(1):277-91.

71. Ramaswamy V, DeSarbo WS, Reibstein DJ, Robinson WT. An empirical pooling approach for estimating marketing mix elasticities with PIMS data. Mark Sci. 1993:12(1):103-24.

72. Ram N, Grimm KJ. Methods and measures: growth mixture modeling: a method for identifying differences in longitudinal change among unobserved groups. Int J Behav Dev. 2009;33(6):565-76.

73. Lo Y, Mendell NR, Rubin DB. Testing the number of components in a normal mixture. Biometrika. 2001:88(3):767-78.

74. McLachlan G, Peel D. Finite mixture models. New Jersey: John Wiley \& Sons; 2004.

75. Leiby BE. Growth curve mixture models. Shanghai Arch Psychiatry. 2012;24(6):355-8.

76. Muthén B, Brown CH, Masyn K, Jo B, Khoo ST, Yang CC, et al. General growth mixture modeling for randomized preventive interventions. Biostatistics. 2002;3(4):459-75.

77. Muthén B, Brown H, Leuchter A, Hunter A. General approaches to analysis of course: applying growth mixture modeling to randomized trials of depression medication. In: Shrout P, editor. Causality and psychopathology: finding the determinants of disorders and their cures. Washington DC: American Psychiatric Publishing; 2009. p. 159-78.

78. Rochat TJ, Bland RM, Tomlinson M, Stein A. Suicide ideation, depression and HIV among pregnant women in rural South Africa. Health (N Y). 2013;5(3A):650-61.

79. Araya R, Rojas G, Fritsch R, Gaete J, Rojas M, Simon G, et al. Treating depression in primary care in low-income women in Santiago, Chile: a randomised controlled trial. Lancet. 2003;361(9362):995-1000.

80. Patel V, Weiss HA, Chowdhary N, Naik S, Pednekar S, Chatterjee S, et al. Effectiveness of an intervention led by lay health counsellors for depressive and anxiety disorders in primary care in Goa, India (MANAS): a cluster randomised controlled trial. Lancet. 2010;376(9758):2086-95.

81. Zimmerman M, Martinez JH, Friedman M, Boerescu DA, Attiullah N, Toba C. How can we use depression severity to guide treatment selection when measures of depression categorize patients differently? J Clin Psychiatry. 2012;73(10):1287-91

82. Sutter-Dallay A, Cosnefroy O, Glatigny-Dallay E, Verdoux H, Rascle N. Evolution of perinatal depressive symptoms from pregnancy to two years postpartum in a low-risk sample: the MATQUID cohort. J Affect Disord. 2012;139(1):23-9.

83. Gold SM, Enck P, Hasselmann H, Friede T, Hegerl U, Mohr DC, et al. Control conditions for randomised trials of behavioural interventions in psychiatry: a decision framework. Lancet Psychiatry. 2017;4(9):725-32.

\section{Publisher's Note}

Springer Nature remains neutral with regard to jurisdictional claims in published maps and institutional affiliations. 